\title{
Vaccination against type 1 angiotensin receptor prevents streptozotocin-induced diabetic nephropathy
}

\author{
Dan Ding ${ }^{1} \cdot$ Yimei Du ${ }^{1} \cdot$ Zhihua Qiu ${ }^{1} \cdot$ Sen Yan $^{2} \cdot$ Fen Chen ${ }^{1} \cdot$ Min Wang ${ }^{1}$.

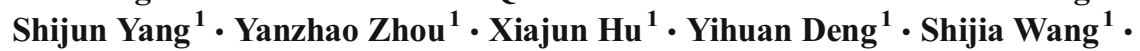 \\ Liangping Wang ${ }^{1} \cdot$ Hongrong Zhang ${ }^{1} \cdot$ Hailang $\mathrm{Wu}^{1} \cdot$ Xian $\mathrm{Yu}^{1} \cdot$ Zihua Zhou $^{1}$. \\ Yuhua Liao ${ }^{1}$ Xiao Chen ${ }^{1}$
}

Received: 30 July 2015 /Revised: 8 September 2015 / Accepted: 16 September 2015 / Published online: 26 September 2015

(C) The Author(s) 2015. This article is published with open access at Springerlink.com

\begin{abstract}
Recently, our group has developed a therapeutic hypertensive vaccine against angiotensin (Ang) II type 1 receptor (AT1R) named ATRQ $\beta-001$. To explore its potential effectiveness on streptozotocin-induced diabetic nephropathy, male Sprague Dawley rats were randomly divided into two groups: a control and a diabetic model. After 1 week, the diabetic rats were divided into four subgroups (each with 15 rats) for 14-week treatments with saline, olmesartan, ATRQ $\beta-001$, and $Q \beta$ virus-like particle (VLP), respectively. In addition to lower blood pressure, ATRQ $\beta-001$ vaccination ameliorated biochemical parameter changes of renal dysfunction, mesangial expansion, and fibrosis through inhibiting oxidative stress, macrophage infiltration, and proinflammatory factor expression. Furthermore, ATRQ $\beta-001$ vaccination suppressed renal Ang II-AT1R activation and abrogated the downregulation of angiotensin-converting enzyme 2-Ang (1-7), similar to olmesartan treatment, while no obvious feedback activation of circulating or local renin-angiotensin system (RAS) was
\end{abstract}

Dan Ding, Yimei Du and Zhihua Qiu contributed equally to this work.

Electronic supplementary material The online version of this article (doi:10.1007/s00109-015-1343-6) contains supplementary material, which is available to authorized users.

Xiao Chen

skycreeper@126.com

1 Laboratory of Cardiovascular Immunology, Key Laboratory of Molecular Targeted Therapies of the Ministry of Education, Institute of Cardiology, Union Hospital, Tongji Medical College of Huazhong University of Science and Technology, Wuhan 430022, China

2 Fuwai Hospital, Peking Union Medical College and Chinese Academy of Medical Sciences, Beijing, China only observed in vaccine group. In rat mesangial cells, the anti-ATR-001 antibody inhibited high glucose-induced transforming growth factor- $\beta 1$ (TGF)- $\beta 1 /$ Smad 3 signal pathway. Additionally, no significant immune-mediated damage was detected in vaccinated animals. In conclusion, the ATRQ $\beta-001$ vaccine ameliorated streptozotocin-induced diabetic renal injury via modulating two RAS axes and inhibiting TGF- $\beta 1 /$ Smad3 signal pathway, providing a novel, safe, and promising method to treat diabetic nephropathy.

\section{Key messages}

- Overactivation of RAS plays a crucial role in the development of the DN.

- Our aim was to verify the effectiveness of ATRQ $\beta-001$ vaccine in STZ-induced DN.

- The ATRQ $\beta-001$ modulated two RAS axes and inhibited TGF- $\beta 1 /$ Smad3 signal pathway.

- The vaccine therapy may provide a novel, safe, and promising method to treat DN.

Keywords Angiotensin receptor · Streptozotocin ·

Renin-angiotensin system · Diabetic nephropathy $\cdot$ Vaccine

\section{Introduction}

Diabetic nephropathy (DN) is the most common cause of endstage renal diseases, responsible for over $40 \%$ of all cases in the USA, and this number is likely to increase unabated [1]. Overactivation of the renin-angiotensin system (RAS) plays a crucial role in the development of the disease [2]. Current treatment, aimed at slowing progression, concentrates on two inter-related therapeutic strategies: blood pressure reduction and blockade of the RAS [3-5]. Such treatments have been shown to reduce the functional changes seen in $\mathrm{DN}$ 
and also to attenuate the structural abnormalities that characterize this disease [6-8]. At present, blockade of the RAS is achieved by two major drug classes: angiotensin-converting enzyme (ACE) inhibitors and angiotensin II (Ang II) receptor blockers (ARBs), agents that also lower systemic blood pressure [9]. However, they are not completely effective in preventing or reversing the progress of $\mathrm{DN}$, in part due to a compensatory increase in plasma renin activity (PRA) or Ang II [10]. Current therapies are insufficient, necessitating the search for new therapeutic strategies in DN.

Recently, we developed a therapeutic hypertensive vaccine ATRQ $\beta-001$, a peptide (ATR-001) derived from human Ang II receptor type 1 (AT1R) conjugated with $\mathrm{Q} \beta$ bacteriophage virus-like particles, which decreased the blood pressure of hypertensive animals effectively through diminishing the pressure response and inhibiting signal transduction initiated by Ang II with no obvious feedback activation of circulating or local RAS [11]. In our previous study, we demonstrated that an epitope from the rat AT1R, designated as ATR12181, could decrease SBP of spontaneously hypertensive rats (SHRs) and provide excellent protections in target organs [12]. Following our work, Hiroshi Itoh and his colleagues emphasized vaccination against AT1R for the prevention of L-NAME-induced nephropathy in SHRs, not only for the attenuation of hypertension [13]. Ang II and other components of RAS also have a central role in the pathogenesis and progression of diabetic renal injuries. Accordingly, this study was designed to explore the possibility of the ATRQ $\beta-001$ vaccine in ameliorating of experimental DN and the potential mechanisms. The study was undertaken in Sprague Dawley rats treated with streptozotocin that developed renal injury similarities to human DN [14].

\section{Methods}

Detailed methods are available in the Online Supplement.

\section{Animals}

Male Sprague Dawley rats weighing 200-250 g were purchased from the experimental animal research center (Hubei Province, China). All animals were kept in the pathogen-free room in the experimental animal center (Tongji Medical College of Huazhong University of Science and Technology, Wuhan, China), and all experiments were carried out in accordance with guidelines for the Care and Use of Laboratory Animals (Science and Technology Department of Hubei Province, China, 2005). Experimental diabetes was induced by intraperitoneal injection of the $\beta$ cell toxin streptozocin $(60 \mathrm{mg} / \mathrm{kg})$ dissolved in fresh sodium citrate buffer ( $\mathrm{pH} 4.5)$ following an overnight fast. Animals with plasma glucose concentrations in excess of $16.7 \mathrm{mmol} / \mathrm{l}, 1$ week postinduction of diabetes, were included in the study. Sham-injected control animals (sodium citrate buffer, $\mathrm{pH} 4.5$ ) were followed concurrently. Diabetes rats were then randomized into four groups ( $n=15)$, receiving one of the following treatments: (1) DN group: equal volume saline injection subcutaneously (s.c); (2) OM group: olmesartan, $5 \mathrm{mg} / \mathrm{kg} / \mathrm{day}$ via oral gavage; (3) ATRQ $\beta$-001group: the ATRQ $\beta-001$ vaccine, which was immunized s.c $400 \mu \mathrm{g}$ on days 0,14 , and 21 , formulated in aluminum hydroxide gel; (4) virus-like particle (VLP) group: $400 \mu \mathrm{g} Q \beta$ VLP injected as group 3. Each week, rats were weighed and their blood glucose levels were measured. ATR-001-specific antibody titers were detected in every 2 weeks. Every 4 weeks, systolic blood pressure (SBP) was determined in preheated conscious rats via tail-cuff plethysmography using a non-invasive blood pressure controller and PowerLab system.

\section{Plasma renin activity, Ang II, and Ang (1-7) concentration measurement}

The rats were decapitated between 9 am and $12 \mathrm{am}$. Blood samples were collected and divided into two parts: one was mixed with the enzyme inhibitor mixture $(1 \mathrm{ml}$ blood in $50 \mu \mathrm{l}$ inhibitor mixture including $20 \mu \mathrm{l} 0.3 \mathrm{~mol} / \mathrm{l}$ EDTA, $10 \mu \mathrm{l}$ $0.32 \mathrm{~mol} / 1 \mathrm{dimercaprol}$, and $20 \mu \mathrm{l} 0.34 \mathrm{~mol} / 1 \mathrm{8}-\mathrm{OH}-$ quinoline sulfate) for PRA and Ang II concentration measurement by radioimmunoassay (RIA) according to the assay kit instruction (NIBT, Beijing); the other was used for biochemical experiments. For tissue Ang II and Ang (1-7) measurement, immediately after harvesting and weighing, the kidney cortex were immersed in ice-cold methanol, minced, and homogenized with tissue homogenizers. The homogenates were centrifuged (4000 rpm, $4{ }^{\circ} \mathrm{C}, 15 \mathrm{~min}$ ), and the supernatants were dried overnight in a vacuum centrifuge. The dried residue was reconstituted in $1 \mathrm{ml}$ RIA buffer and then was subjected to HPLC to separate Ang II from other substances. The tissue Ang II concentration was detected according to the assay kit instruction (NIBT, Beijing), and Ang (1-7) was measured by HPLC.

\section{Biochemical measurement}

The plasma samples were used for the measurement of glucose, lipid level, creatinine $(\mathrm{Cr})$, and blood urea nitrogen (BUN). Urine samples were collected using metabolic cages, and the supernatant was used for examination of the 24-h urinary protein.

\section{Histopathology}

Parts of fresh left renal cortex were immediately fixed in $0.25 \%$ glutaraldehyde for transmission electron microscopy (TEM). The other parts were fixed with $4 \%$ 
paraformaldehyde overnight, embedded in paraffin for histopathology. Sections were stained with hematoxylin-eosin (H\&E), Masson's trichrome, and periodic acid-Schiff (PAS). Frozen sections were stained with dihydroethidium (DHE).

\section{Immunohistochemistry}

Immumohistochemical staining was performed to detect the expression of TGF- $\beta 1$ (1:100, Everest Biotech) and macrophages (CD68, 1:100, AbD Serotec) in glomeruli.

\section{Immunofluorescence}

Immunofluorescence staining of nephrin and podocin expression was performed on the paraffin sections of kidneys using monoclonal anti-nephrin (1:200, Abcam) and anti-podocin antibodies (1:200, Abcam).

\section{Cell culture and treatment}

Rat mesangial cells (RMCs) were cultured in Dulbecco's modified Eagle's medium supplemented with $10 \%$ fetal bovine serum, $100 \mathrm{U} / \mathrm{ml}$ penicillin, and $100 \mathrm{mg} / \mathrm{ml}$ streptomycin at $37{ }^{\circ} \mathrm{C}$ in $95 \%$ air and $5 \% \mathrm{CO}_{2}$. Cells plated on $60-\mathrm{mm}$ dishes were cultured to $80 \%$ confluence and divided into five groups: control group, in which cells were incubated in $5 \mathrm{mmol} / \mathrm{l} \mathrm{D}$-glucose DMEM, and $20 \mathrm{mmol} / \mathrm{l} \mathrm{D}$-mannitol was added to the medium in order to take into the account of the effect of high osmolarity in other cell groups; HG group, in which cells were stimulated with a high concentration of glucose (25 mmol/l) only for $24 \mathrm{~h}$; Los group, in which cells were pretreated with $10^{-6} \mathrm{~mol} / \mathrm{l}$ losartan for $1 \mathrm{~h}$; anti-ATR-001 group, in which cells were pretreated with anti-ATR-001 for $1 \mathrm{~h}$; anti-NATR-001 group, in which cells were pretreated with anti-NATR-001 for $1 \mathrm{~h}$. All cells except control group received stimulation with a high concentration of glucose ( $25 \mathrm{mmol} / \mathrm{l})$ for $24 \mathrm{~h}$ after treatment. Cell protein and messenger RNA (mRNA) were extracted for Western blot and qRTPCR analysis.

\section{Statistical analysis}

Data were shown as the mean \pm SEM. Statistical analyses of the data were performed with one-way ANOVA using SPSS18.0. $P<0.05$ was considered statistically significant.

\section{Results}

\section{Animal characteristics}

In comparison with control animals, diabetic rats had significantly reduced body weight, which was unaffected by treatment. The ratio of kidney weight/body weight was increased, while ATRQ $\beta-001$ vaccine and olmesartan treatments decreased the level. Blood glucose and lipid levels were elevated to a similar extent in all diabetic rat groups, irrespective of treatment (Table 1). No evidence of skin damages at the site of subcutaneous injection was noted in vaccine-treated animals. Less activities and poorer hair were shown in diabetic rats, but the reactivity was the same as normal rats.

\section{ATRQ $\beta-001$ vaccination effectively reduced blood pressure and reversed biochemical parameters of renal dysfunction in STZ-induced diabetic rats}

To examine antibody production, we measured the amounts of anti-ATR-001 antibodies produced in response to vaccination

Table 1 Animal characteristics

\begin{tabular}{lcccll}
\hline & Con & DN & VLP & OM & ATRQß-001 \\
\hline Body weight $(\mathrm{g})$ & $359 \pm 12.69$ & $173.3 \pm 9.78^{*}$ & $169.2 \pm 8.47^{*}$ & $216 \pm 18.68^{*}$ & $184.7 \pm 9.7^{*}$ \\
Kidney weight $(\mathrm{g})$ & $1.19 \pm 0.03$ & $1.15 \pm 0.03$ & $1.14 \pm 0.02$ & $1.36 \pm 0.08$ & $1.11 \pm 0.04$ \\
KW/BW (\%) & $0.33 \pm 0.01$ & $0.67 \pm 0.02^{*}$ & $0.69 \pm 0.02^{*}$ & $0.58 \pm 0.02^{*} \&$ & $0.63 \pm 0.03^{*}$ \\
Blood glucose (mmol/l) & $7.8 \pm 1.84$ & $31.6 \pm 3.11^{*}$ & $34.0 \pm 1.84^{*}$ & $30.4 \pm 1.30^{*}$ & $30.2 \pm 2.55^{*}$ \\
TC (mmol/l) & $1.49 \pm 0.1$ & $2.48 \pm 0.56^{*}$ & $2.6 \pm 0.63^{*}$ & $2.33 \pm 0.16^{*}$ & $2.58 \pm 0.34^{*}$ \\
TG (mmol/l) & $0.85 \pm 0.19$ & $2.53 \pm 0.28^{*}$ & $2.32 \pm 0.17^{*}$ & $2.19 \pm 0.37^{*}$ & $2.31 \pm 0.28^{*}$ \\
LDL-C (mmol/l) & $2.87 \pm 0.44$ & $4.48 \pm 0.65^{*}$ & $4.21 \pm 0.71^{*}$ & $4.09 \pm 0.75^{*}$ & $4.01 \pm 0.91^{*}$ \\
HDL-C (mmol/l) & $0.77 \pm 0.04$ & $0.81 \pm 0.13$ & $0.79 \pm 0.19$ & $0.96 \pm 0.11$ & $0.98 \pm 0.05$ \\
\hline
\end{tabular}

Values are mean \pm SEM

$K W / B W$ kidney weight/body weight, $T C$ total cholesterol, $T G$ total triglyceride, $L D L$ - $C$ low-density lipoprotein cholesterol, $H D L-C$ high-density lipoprotein cholesterol, Con control group, $D N$ diabetes with saline treatment, $V L P$ diabetes with Q $\beta$ VLP treatment, $O M$ diabetes with olmsartan treatment, $A T R Q \beta-001$ diabetes with the ATRQ $\beta-001$ vaccine treatment

$* P<0.05$ vs. Con; $\# P<0.05$ vs. DN; $\& P<0.05$ vs. VLP 
with ATRQ $\beta-001$. After the second injection of the vaccine, the ATR-001-specific antibody titer was 1:10,000 to 1:30,000, and it rose after the third injection, then peaked on day 42 $(1: 40,000)$ and gradually decreased thereafter (Fig. 1a). To investigate the efficacy in blood pressure, systolic blood pressure (SBP) levels were measured by the tail-cuff method. Over the course of the study, SBP was elevated in DN compared to normal rats, while rats immunized with the ATRQ $\beta-001$ vaccine were decreased compared to the VLP group, with a maximum decrease of $19.4 \mathrm{mmHg}(129.9+2.9$ vs. $149.3+$ $3.6 \mathrm{mmHg}, P<0.01$, Fig. 1b).

To evaluate the biochemical parameters of renal function, $24 \mathrm{~h}$ urine volume, and total protein excretion, blood urea nitrogen and creatinine were measured. Diabetic rats exhibited higher levels in all these parameters. ATRQ $\beta$ 001 vaccine and olmesartan-treated rats significantly reduced these parameters compared to DN and VLP groups, and no significant differences were between these two groups (Fig. 1c-f).

\section{ATRQ $\beta-001$ vaccination prevented podocyte injury and loss as well as mesangial expansion}

To confirm the effect of vaccination, we evaluated renal pathological changes. Podocytes form the filtration slit diaphragms that prevents the escape of plasma protein from the glomerular circulation. The disruption of podocytes contributes to proteinuria and further development of glomerular sclerosis [15]. Reduced nephrin and podocin immunostaining in glomeruli were showed in diabetic rats, and ATRQ $\beta-001$ vaccination prevented the reduction (Fig. 2).

In addition to podocyte injury, glomerular mesangial expansion is also a hallmark of DN. Extracellular matrix deposition was detected by periodic acid-Schiff staining, and glomerular sclerosis index (GSI) was calculated. Compared with DN and VLP groups, GSI was significantly decreased in ATRQ $\beta$-001-vaccinated group, similar with olmesartantreated group, which was also confirmed by transmission electron microscopy (TEM) (Fig. 2).
Fig. 1 Antibody titers, systolic blood pressure, and biochemical parameters. a Diabetic rats were immunized on days $0,14,21$, and 70, and the ATR-001-specific antibody titers were screened on days $21,28,42,56,70,77,91$, and 98 . b Systolic blood pressure on weeks $14 ; * P<0.05$ vs. the control group; $\# P<0.05$ vs. DN group; $\& P<0.05$ vs. VLP group. c 24 h urine volume; $* P<0.05$ vs. the control group at the same time point. d $24 \mathrm{~h}$ urine protein; ${ }^{*} P<0.05$ vs. the control group at the same time point; $\# P<0.05$ vs. $\mathrm{DN}$ group at the same time point; $\& P<0.05$ vs. VLP group at the same time point. e Plasma creatinine level $(n=10)$. f Blood urea nitrogen level $(n=10)$. $* P<0.05$ vs. control group; $\# P<0.05$ vs. DN group; $\& P<0.05$ vs. VLP group
A

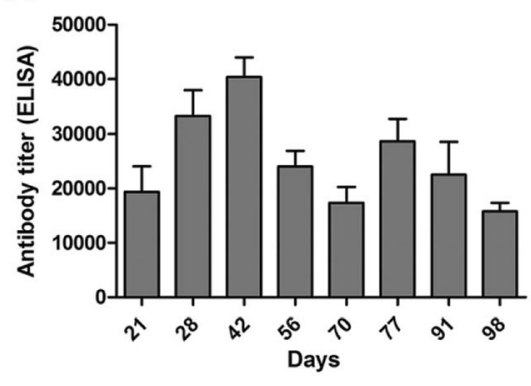

C

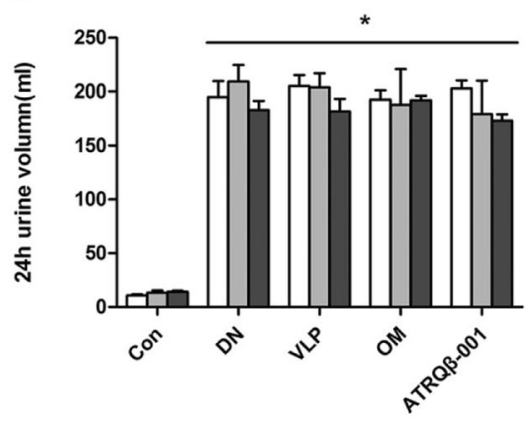

E

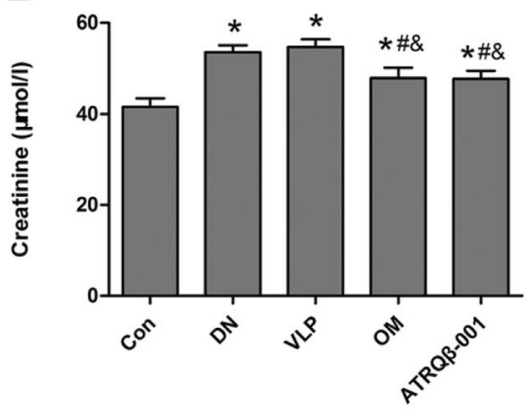

B

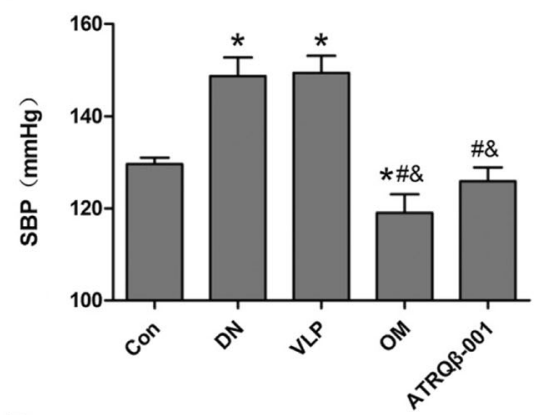

D

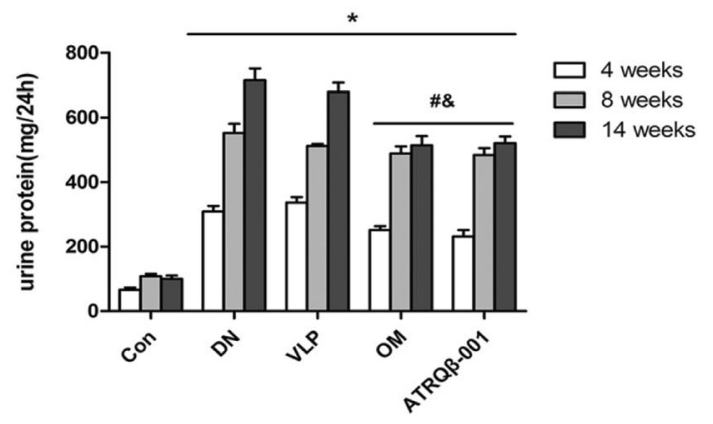

$\mathbf{F}$

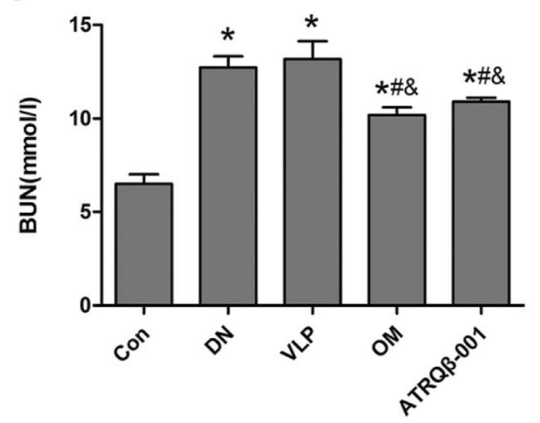


A

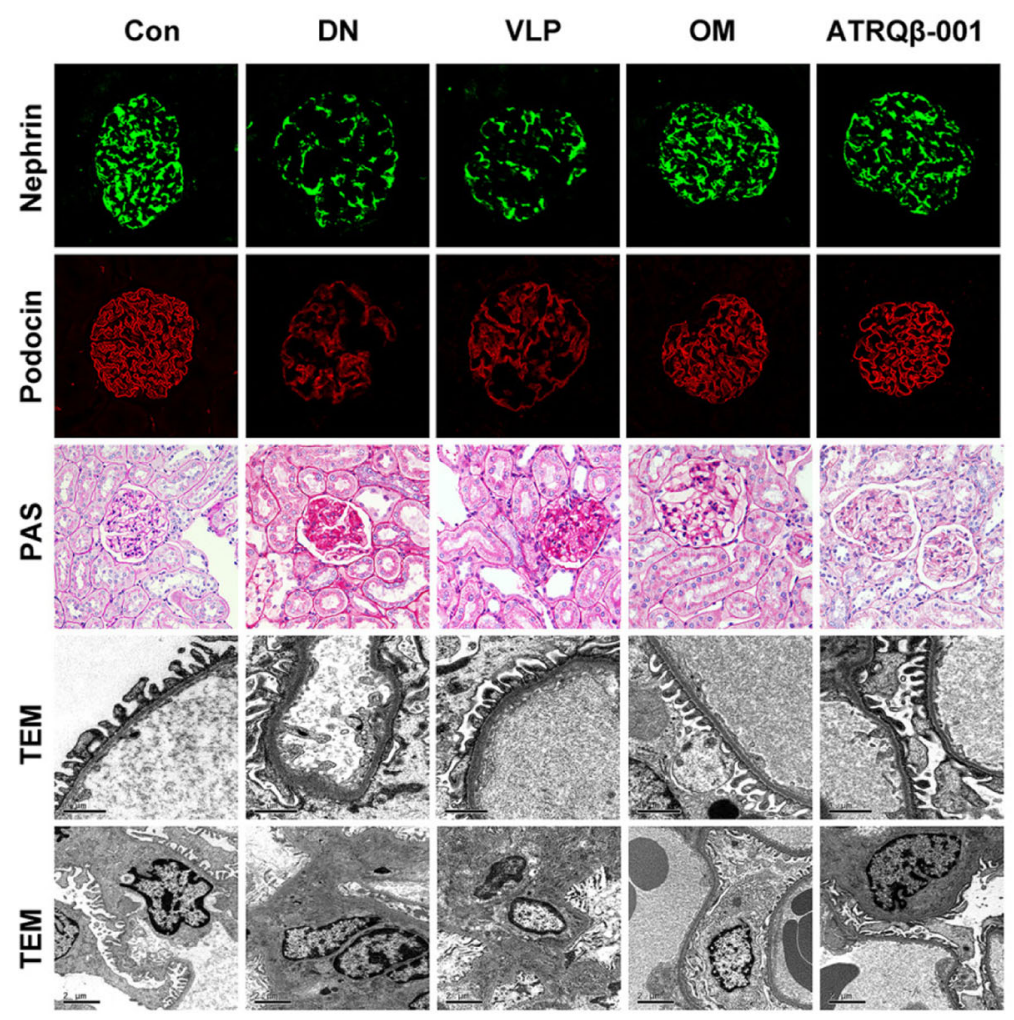

B

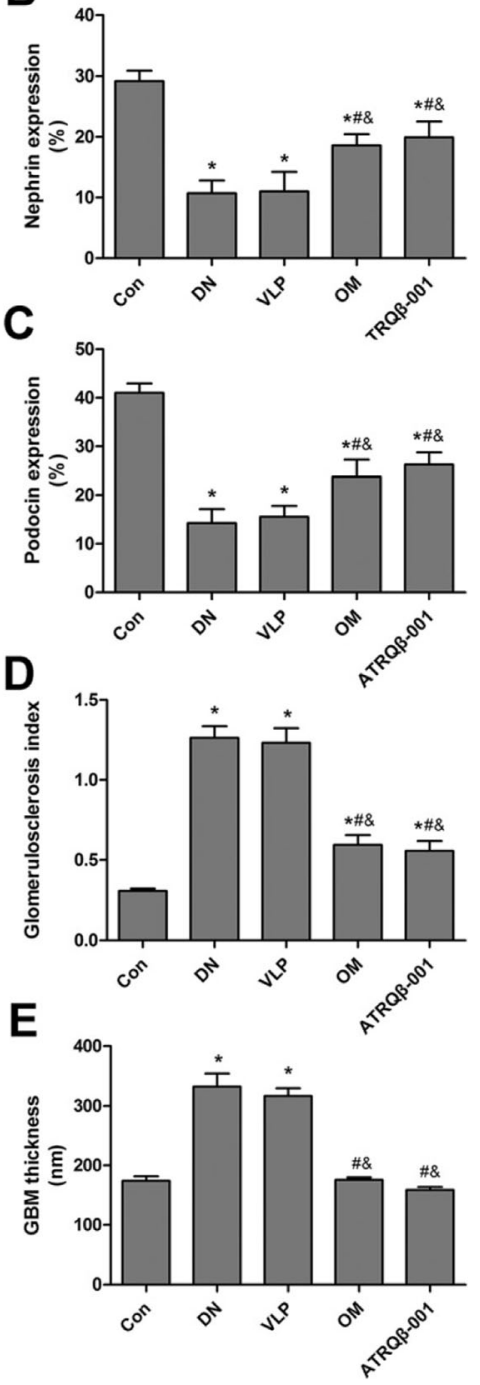

Fig. 2 ATRQ $\beta-001$ vaccination prevented podocyte injury and loss as well as mesangial expansion. a Representative micrographs of nephrin and podocin expression, periodic acid-Schiff (PAS) staining (400×), and transmission electron microscopy (TEM). TEM showed the glomerular basement membrane and the mesangial area. b Quantitative analysis of nephrin expression. c Quantitative analysis of podocin expression. d Quantitative analysis of glomerular sclerosis index based on PAS staining. e Quantitative analysis of glomerular basement membrane thickness. ${ }^{*} P<0.05$ vs. control group; $\# P<0.05$ vs. $D N$ group; $\& P<0.05$ vs. VLP group

\section{ATRQ $\beta-001$ vaccination ameliorated renal fibrosis and inflammation}

To further investigate the mechanisms, renal fibrosis and inflammation parameters were measured. The extent of interstitial expansion was quantified by Masson's trichrome-stained sections. The interstitial expansive index was higher in DN and VLP groups, and ATRQ $\beta-001$ vaccination blocked the increase (Fig. 3). Glomerular and interstitial inflammation was quantified by the cell numbers of macrophages stained positively with CD68 antibody, and ATRQ $\beta-001$ vaccination successfully prevented the increased inflammation (Fig. 3). Besides, we detected transforming growth factor- $\beta 1$ (TGF- $\beta 1$ ) expression and reactive oxygen species, which was also decreased by ATRQ $\beta-001$ vaccine treatment. Further, the mRNA expressions of profibrotic and proinflammatory factors in kidney cortex were measured. As shown in Fig. 3f, g, the increased mRNA levels in these factors were significantly suppressed by ATRQ $\beta-001$ vaccination. There were no significant differences between ATRQ $\beta-001-$ and olmesartan-treated groups.

ATRQ $\beta-001$ vaccination suppressed renal Ang II-AT1R activation and abrogated the downregulation of ACE2-Ang (1-7) with no feedback of RAS

To determine whether blockade of AT1R lead to feedback activation of circulating RAS, we detected the 
A

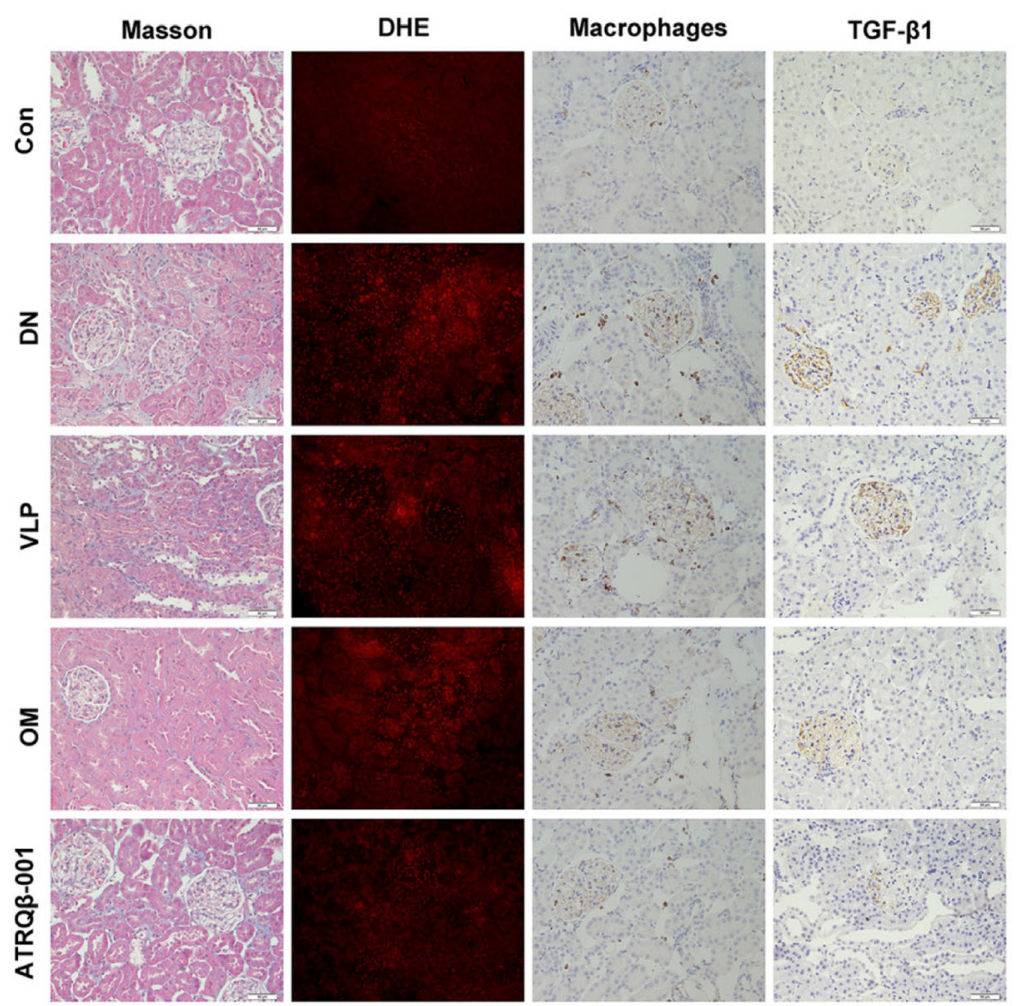

$\mathbf{F}$

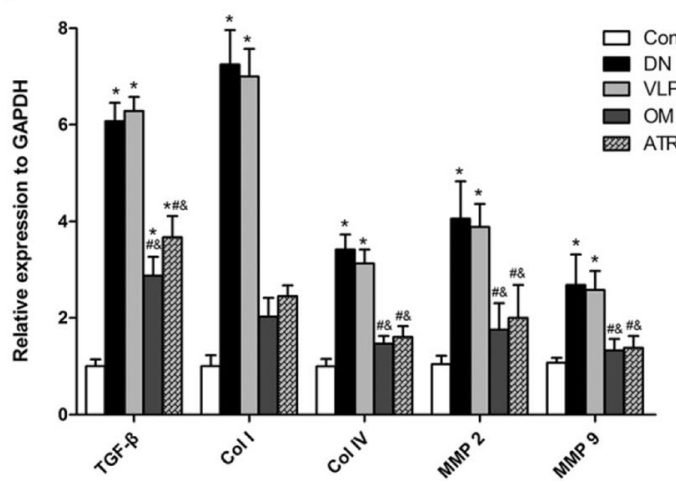

Fig. 3 ATRQ $\beta-001$ vaccination ameliorated renal fibrosis and inflammation. a Representative staining of Masson's trichrome, DHE $(200 \times)$, macrophages, and TGF- $\beta 1(400 \times)$. b Quantitative analysis of interstitial fibrosis. c Quantitative analysis of DHE fluorescence. d Quantitative analysis of glomerular and interstitial macrophage

PRA and Ang II concentration. The PRA in ATRQ $\beta$ 001 vaccinated group was $13660 \pm 2904 \mathrm{pmol} / \mathrm{h} / \mathrm{l}$, which had no significant difference with VLP group $(21,450 \pm 2851 \mathrm{pmol} / \mathrm{h} / \mathrm{l}, P=0.48)$. However, a distinct increase was observed in olmesartan group (43 $040 \pm 8$ $157 \mathrm{pmol} / \mathrm{h} / \mathrm{l}, P=0.005$; Fig. 4a). Similarly, the plasma
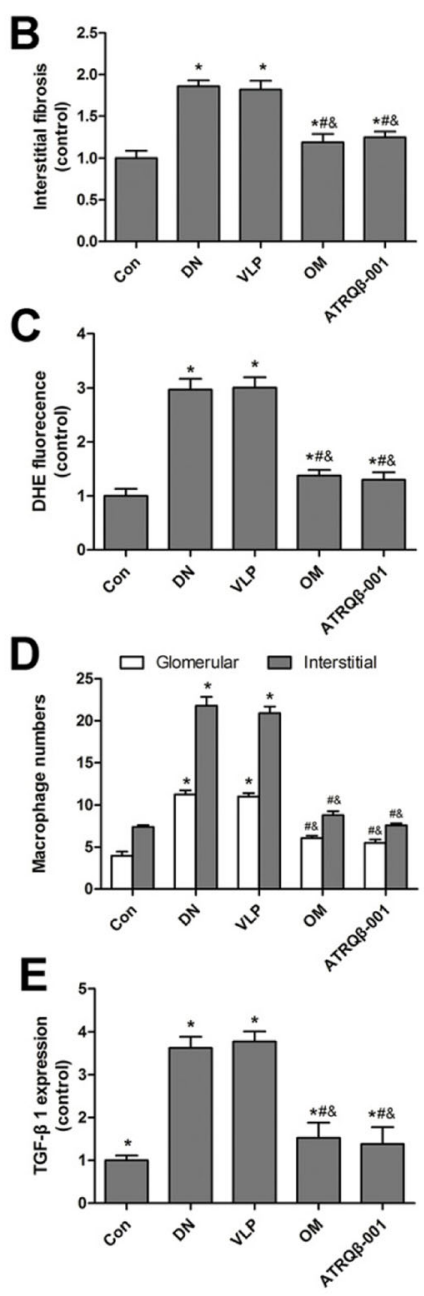

G

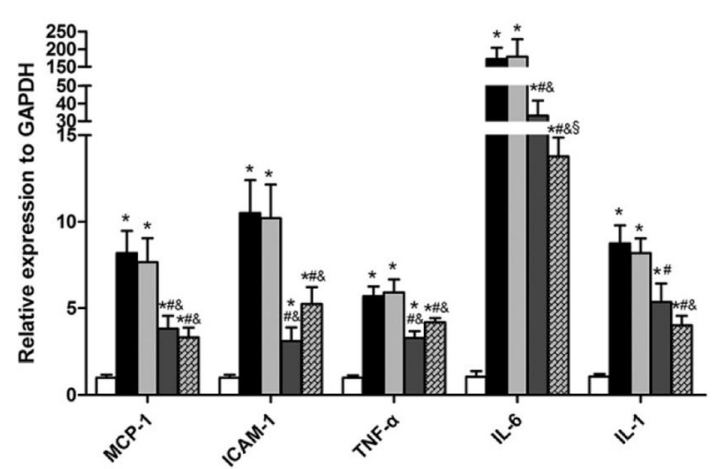

numbers. e Quantitative analysis of TGF- $\beta 1$ expression. $\mathbf{f}$ The levels of profibrotic factor mRNA expression measured by quantitative real-time PCR $(n=10)$. $g$ The levels of proinflammatory factor mRNA expression measured by quantitative real-time PCR $(n=10)$. ${ }^{*} P<0.05$ vs. control group; $\# P<0.05$ vs. DN group; $\& P<0.05$ vs. VLP group

concentration of Ang II in olmesartan group was higher than VLP group $(567.3 \pm 80.7$ vs. $291.8 \pm 29.16 \mathrm{pmol} / 1$, $P=0.02$ ), whereas no significant difference was observed between VLP and vaccine groups $(291.8 \pm 29.16$ vs. $288.7 \pm 19.33, P=0.53$; Fig. $4 b$ ). To further examine the local RAS, kidney Ang II concentration was 

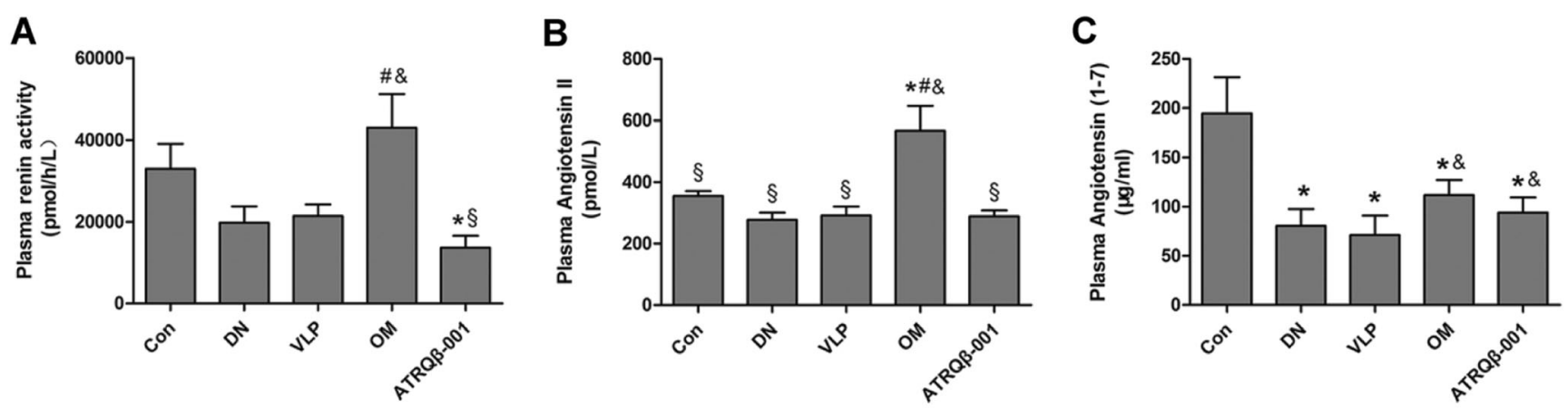

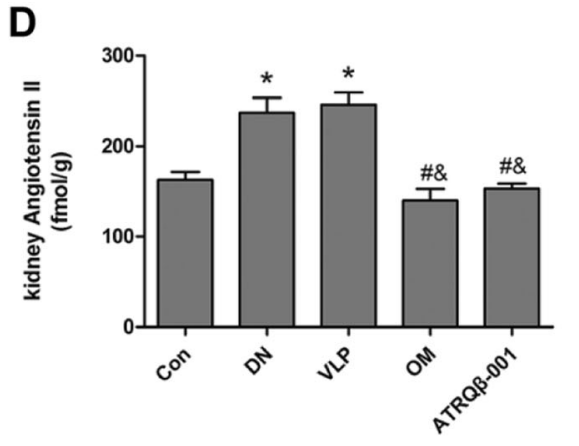

Fig. 4 Circulating or local RAS were not activated in ATRQ $\beta-001-$ vaccinated diabetic rats. a Plasma renin activity. b Plasma angiotensin II concentration. c Plasma angiotensin (1-7) concentration. d kidney

measured. The concentration of Ang II in kidneys was lower in olmsartan- and vaccine-treated groups compared to DN and VLP groups (Fig. 4d). To investigate whether the protection effect was a result of increase Ang (1-7) activation with simultaneous Ang II suppression, we subsequently measured plasma and kidney Ang (1-7). The decline of Ang (1-7) in diabetic rats was improved in ATRQ $\beta-001$ vaccine and olmesartantreated groups (Fig. 4c, e).

Activation of RAS in streptozotocin (STZ)-induced diabetic kidneys was further confirmed by Western blot and quantitative real-time PCR (Fig. 5). The mRNA expression of kidney renin in olmesartan group was obviously higher than VLP group $(P<0.001)$, while no difference was found between ATRQ $\beta-001$ vaccine and VLP groups (Fig. $5 \mathrm{~g}$ ). $\mathrm{ACE}$ and AT1R expressions were increased in diabetic rats, and both ATRQ $\beta-001$ vaccine and olmesartan groups attenuated the increasing expressions. ACE2-Ang (1-7)-mas axis counteract Ang II-mediated effects in various organs, including the kidneys, and we observed reduced expression of ACE2-Ang (1-7)-mas in the diabetic kidneys. ATRQ $\beta-001$ vaccine and olmesartan displayed upregulation of ACE2Ang (1-7)-mas expression compared with VLP group. Further, we also detected the downstream signal transduction and the activation of ERK1/2, and p38 MAPK phosphorylation was inhibited by ATRQ $\beta-001$ vaccination, similar to olmesartan treatment (Fig. 5e-f).

\section{Anti-ATR-001 inhibited the TGF- $\beta 1 /$ Smad3 signal pathway and expression of collagen IV and fibronectin in rat mesangial cells}

To further explore the mechanisms, cell experiment was undertaken by anti-ATR-001 stimulation. Glomerular mesangial cells are believed to be responsible for overproduction of ECM proteins in various pathologic conditions, and TGF- $\beta 1$ has been proposed to play an important role. The TGF- $\beta 1 /$ Smad3 signal pathway regulates the hypertrophic and prosclerotic changes in diabetic renal injury [16]. Anti-ATR001 and losartan treatment significantly decreased the expression of TGF- $\beta 1$ and the phosphorylation levels of Smad 3 in RMCs stimulated by high glucose. Additionally, we examined the expression of collagen IV and fibronectin, which showed similar change as TGF- $\beta 1$ (Fig. 6).

\section{No imumune-mediated injury was observed in vaccinated animals}

For safety consideration, normal SD rats were immunized with the ATRQ $\beta-001$ vaccine, and the histological changes of kidney were observed by light microscopy and TEM. No evidence of skin damages at the site of subcutaneous injection was noted in vaccine-treated animals. Compared with control group, no obvious cell proliferation and pathological changes in the mesangial 
A

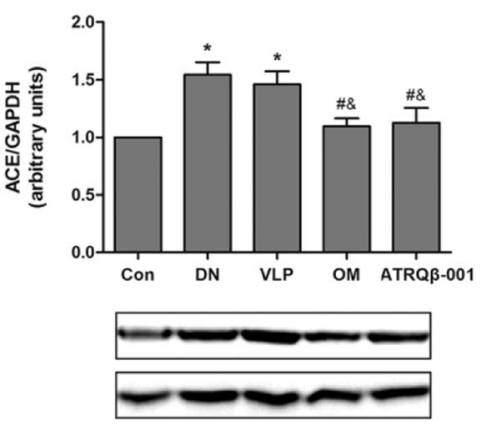

D

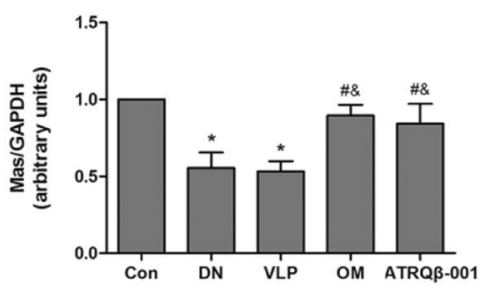

B

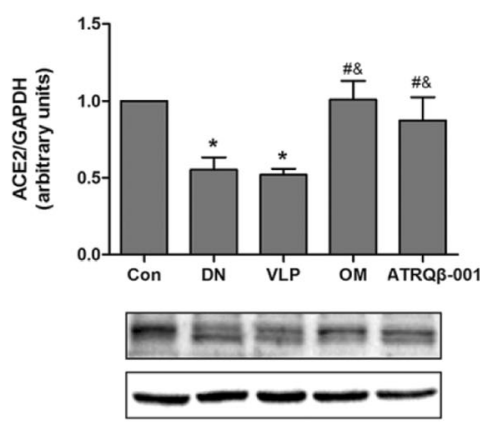

E
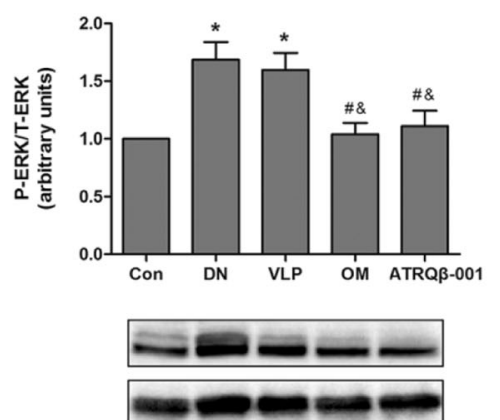

C
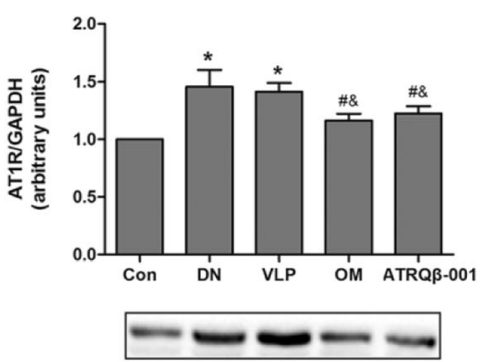

$m-\infty-\infty$

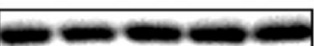

$\mathbf{F}$
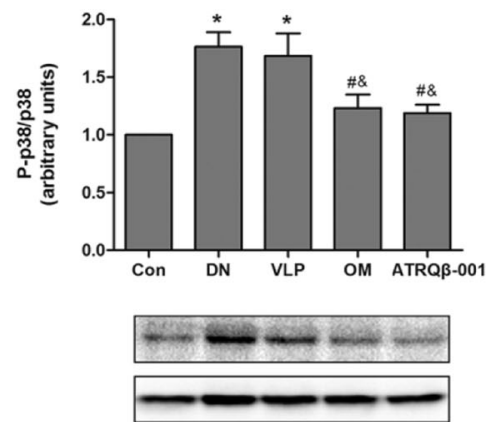

G

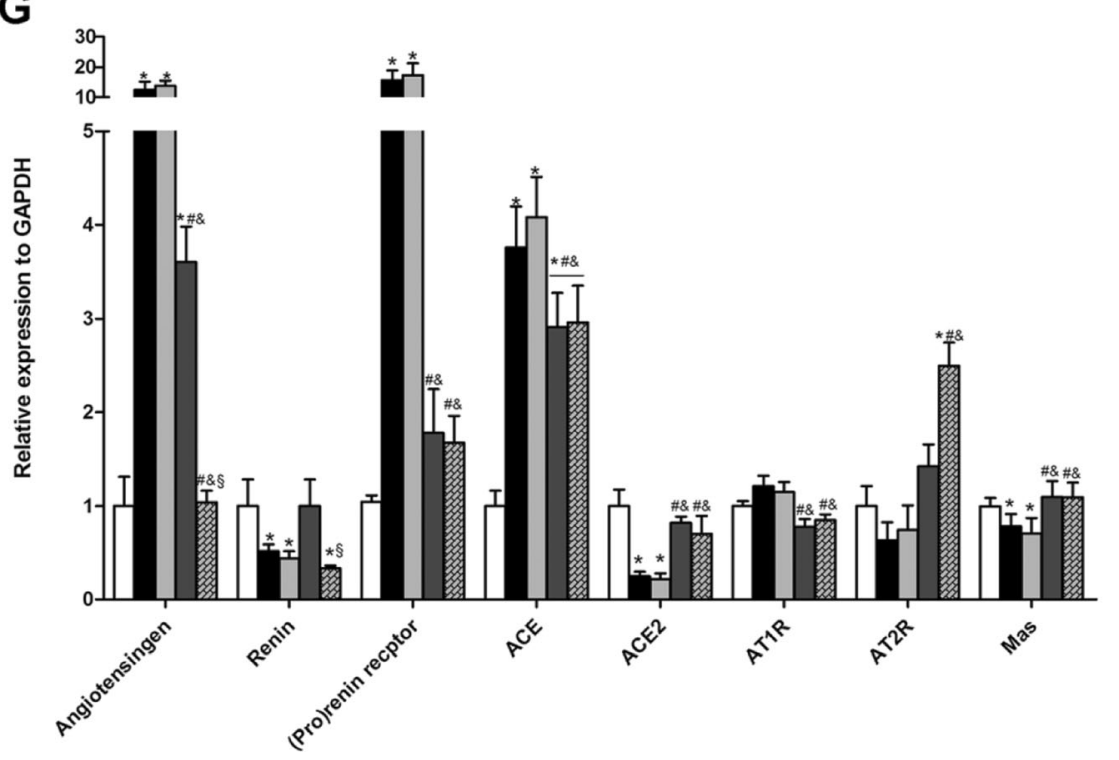

Fig. 5 Effect of ATRQ $\beta-001$ vaccination on the RAS components, ERK1/2, and P38 MAPK in STZ-induced diabetic kidneys. Representative Western blots (lower panel) showing specific bands for a ACE, b ACE2, c AT1R, d Mas, e P-ERK1/2, and f P-p38 and quantitative analysis were presented on upper panel $(n=10)$. $\mathbf{g}$ The

area were shown in vaccine group. Also, TEM demonstrated that no immune complexes were observed in the basement membrane, and the structure of the glomerulus was intact (Fig. 7). relative mRNA expression of angiotensingen, renin, (pro)renin receptor, ACE, ACE2, AT1R, AT2R, and Mas detected by quantitative real-time PCR $(n=10) .{ }^{*} P<0.05$ vs. control group; $\# P<0.05$ vs. DN group; $\& P<0.05$ vs. VLP group

\section{Discussion}

In this study, we demonstrated for the first time that in a rat model of DN, a vaccine targeting AT1R named ATRQ $\beta-001$ 
A

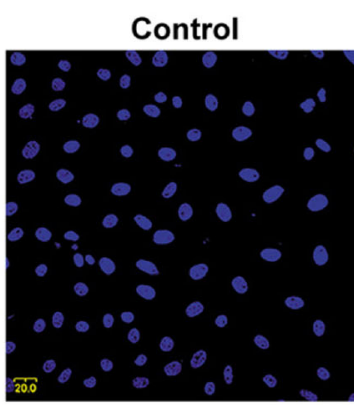

C

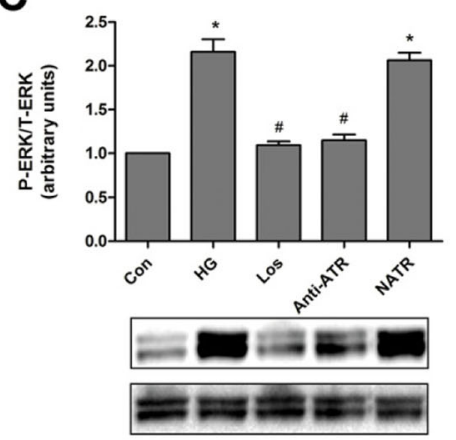

$\mathbf{F}$

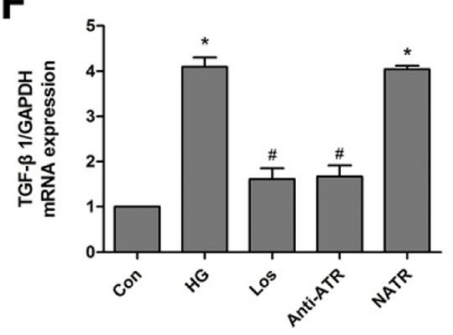

D

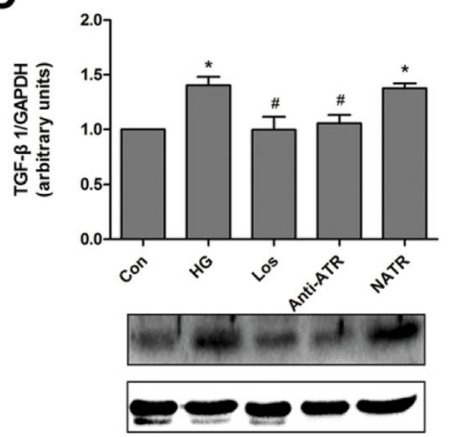

G

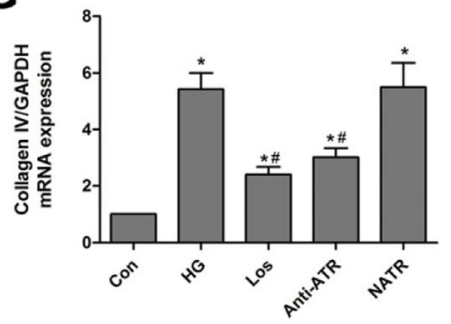

B

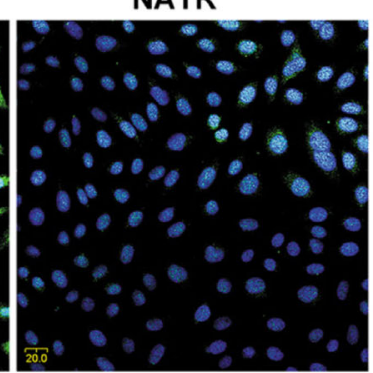

\section{Con Anti-ATR}

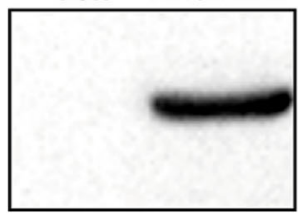

E

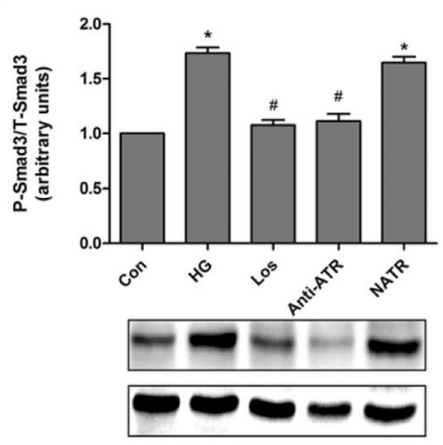

H

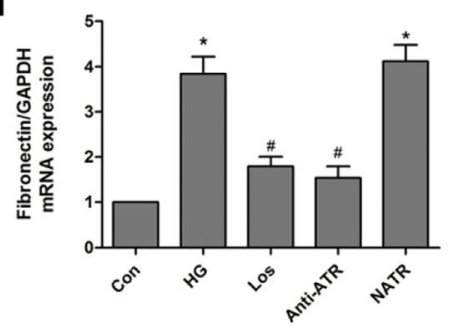

Fig. 6 Anti-ATR-001 inhibited the TGF- $\beta 1 / \mathrm{Smad} 3$ signal pathway and expression of collagen IV and fibronectin in rat mesangial cells. As shown on $\mathbf{a}$ and $\mathbf{b}$, anti-ATR-001 specifically bound to AT1R in rat mesangial cells (RMCs). Representative Western blots (lower panel) showing specific bands for $\mathbf{c}$ P-ERK1/2, d TGF- $\beta 1$, e Smad3, and quantitative analysis were presented on upper panel. The relative expression of TGF- $\beta 1$ and collagen I and IV mRNA were shown on $\mathbf{f}, \mathbf{g}$, and $\mathbf{h}$. Con control, HG high glucose ( $25 \mathrm{mmol} / \mathrm{l})$, Los losartan treatment, Anti-ATR anti-ATR-001 treatment, NATR anti-NATR-001 treatment $(n=5)$. $* P<0.05$ vs. con; $\# P<0.05$ vs. HG attenuated the progression of the disease, as exhibited by reduction of biochemical parameters of renal dysfunction and amelioration of renal pathological changes.

The rat DN model was induced by an intraperitoneal injection of STZ that has been widely used to create diabetes models in rodents with pathological features similar to human diabetic nephropathy [14]. The nephropathy subcommittee of the Animal Models of Diabetic Complications Consortium (AMDCC) has published the following validation criteria for rodent models of DN based on the clinical and pathological features of human DN: (1) $>50 \%$ decrease in renal function, (2) $>10$-fold increase in albuminuria, and (3) pathological features including advanced mesangial matrix expansion ( \pm nodules), thickening of the glomerular basement membrane, arteriolar hyalinosis, and tubulointerstitial fibrosis [17]. An ideal model of DN would display all of these criteria; however, no current model entirely satisfies them. That is the limitation in animal experiments. As the rodent models share many similarities with human disease, we cannot ignore the potential therapeutic value of the vaccine in the treatment of human diseases. Our results showed that rats presented severe hyperglycemia and renal dysfunction manifested as increased plasma creatinine level, BUN, $24 \mathrm{~h}$ urinary volume, and protein excretion, indicating that a DN animal model was successfully established. The ratio of kidney weight/body weight in diabetic groups was significantly increased compared with that in control group, substantial to the presence of renal hypertrophy in diabetic rats. During the course of the study, systolic blood pressure was elevated in diabetic rats, notwithstanding the limitations of tail-cuff sphygmomanometry, ATRQ $\beta-001$ vaccination lowered blood pressure (BP) smoothly, and olmesartan decreased to a greater extend. It is worth noting that our animal 
Fig. 7 No imumune-mediated injury was observed in vaccinated animals. a Representative staining of hematoxylin-eosin (H\&E), Masson's trichrome, and periodic acid-Schiff (PAS) and transmission electron microscopy (TEM). b Normal SD rats were immunized on days 0 and 14, and the ATR-001-specific antibody titers were screened on days 21 , $28,42,56,77,97,120$, and 150
A
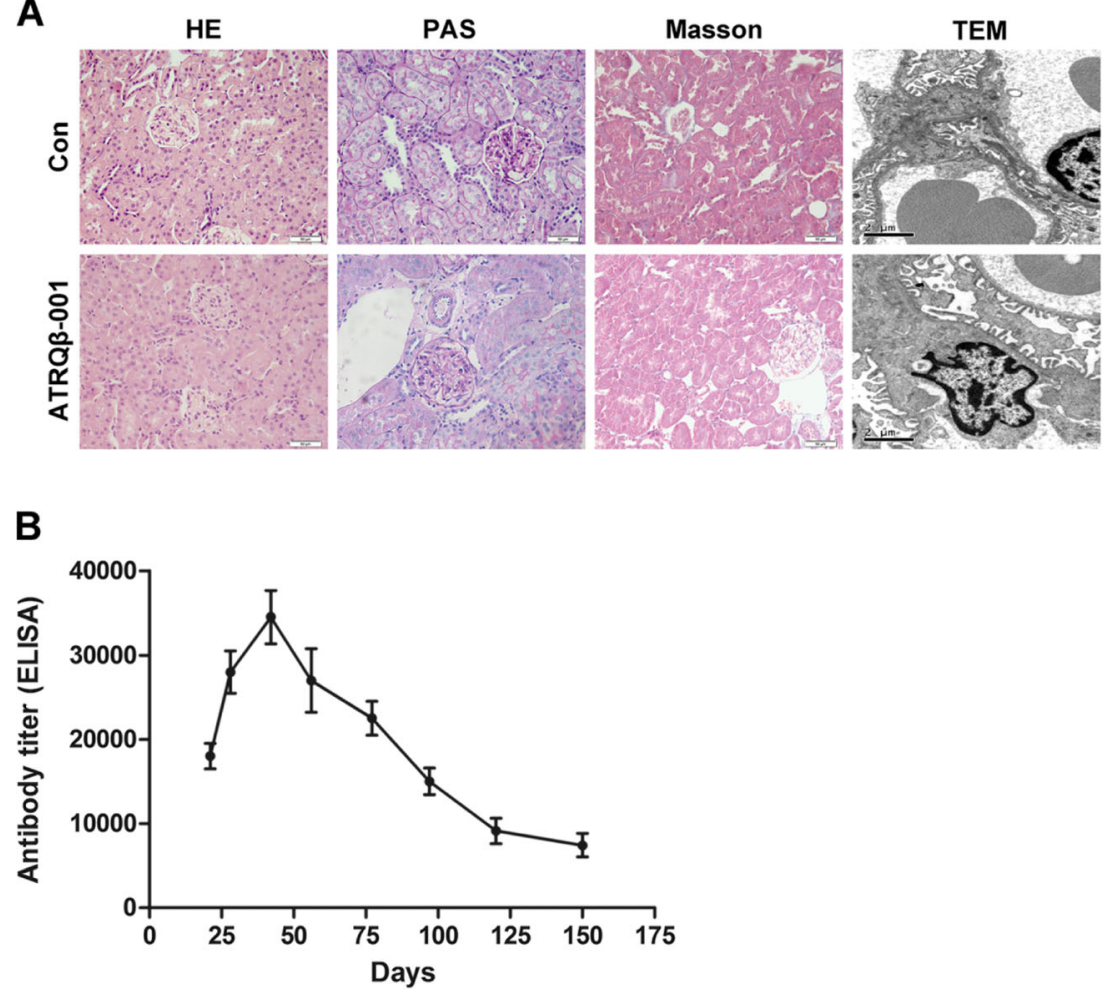

experiments showed many times that the antihypertensive effect of ATRQ $\beta-001$ vaccine was dependent on the baseline of the BP level and exhibited no decreasing effect in normotensive rats. Oppositely, ARBs decreased BP irrespective of the basic level and had an increase risk of hypotension. Therefore, ATRQ $\beta-001$ vaccine may be more practical in diseases where the BP was not very high, such as DN.

Glomerular mesangial matrix expansion, renal fibrosis, and inflammation are typical pathological features of DN. ATRQ $\beta-001$ vaccination ameliorated these morphologic changes of renal injury as well as decreased expression of profibrotic and proinflammatory factors. To further confirm the effects, RMCs were used to investigate the mechanism in vitro. The anti-ATR-001 antibody treatment effectively inhibited high glucose-induced extracellular signal-regulated kinase phosphorylation and TGF- $\beta 1 / \mathrm{Smad} 3$ signal pathway. These results strengthened the renoprotection of ATRQ $\beta-001$ vaccination in addition to the antihypertensive effect. The inhibition of oxidative stress and macrophage infiltration as well as proinflammatory factor expression may contribute to the amelioration of renal pathological changes.

Furthermore, compared with the obvious RAS feedback of ARBs, circulating or local RAS were not elevated in diabetes animals immunized with ATRQ $\beta-001$ vaccine. Nevertheless, kidney Ang II concentration and AT1R expression were decreased in both groups. The recent development of ACE2Ang (1-7)-mas concept has provided new insights into the effects of Ang II in animal experimental models. This novel concept states that RAS has two axes: the ACE-Ang II-AT1R axis and ACE2-Ang (1-7)-mas axis. The former axis induces vasoconstriction, proliferation, and proinflammatory functions through Ang II, and the latter axis counteract the effects of the former axis through the major heptapeptide effector Ang (1-7) $[18,19]$. ACE2 cleaves Ang II to produce abundant levels of Ang (1-7) in the proximal tubule, which produces vasodilation and antiproliferative, natriretic, and diuretic effects [20]. Despite its significant role, the exact role of ACE2 and Ang (1-7) in kidney disease is not clearly understood. It has been reported that inhibition of ACE2 function accelerates diabetic injury and human recombinant ACE2 reduces the progression [21, 22]. Moreover, Ang (1-7) attenuates the progression of diabetic nephropathy in animal models $[23,24]$. Our study showed a downregulation of ACE2-Ang (1-7)-mas axis in diabetic rats, and the beneficial effects of ATRQ $\beta-001$ vaccine and olmesartan treatment may partly due to the upregulation of this axis. Although the exact mechanism of how these two axes counteract with each other remains unknown, we can conclude that the vaccine regulates the two RAS axes, not only inhibiting AT1R overactivation.

Safety considerations are paramount when developing any vaccine but are particularly important when targeting a condition for which many safe and effective alternative therapies are available. Four key factors define the safety of therapeutic vaccine: (1) the targeted molecule, (2) the reversible of antibody response, (3) antibody-dependent cell-mediated cytotoxicity (ADCC) and complement-mediated cytotoxicity, and (4) 
activation of $\mathrm{T}$ cells against self-molecules [25]. Combined with our previous studies, vaccination-targeted AT1R showed no immune-mediated injuries $[11,12]$ and was also confirmed in this study. The antibody response was reversible as the possibility of halting antibody production. For ADCC and complement-mediated cytotoxicity, there are still several controversies in terms of its onset and regulation. Nevertheless, the potential effects caused by the vaccine needed further investigation. As the target peptide of the vaccine was only eight amino acids in length, then it was smaller than the minimal size of a $\mathrm{T}$ cell epitope and therefore should not be able to induce a $\mathrm{T}$ cell response [26]. Several studies had estimated that vaccines comprising self-molecules coupled to VLPs present optical candidates capable of achieving efficacious antibody levels while fulfilling the necessary safety criteria [27, 28]. Examples like the Ang II vaccine CYT-006AngQb, which showed effectively reduced BP without any uncontrolled immune stimulation in both animals and patients $[29,30]$. Similar to the composition, the kidney damage caused by immune complexes was not detected and no visible pathological changes were observed by light microscopy and TEM in ATRQ $\beta-001$-vaccinated animals. From the results above, the ATRQ $\beta-001$ vaccine was found to be basically safe, although further assessments are needed to confirm this conclusion.

Compared with chemical drugs, the vaccine therapy has potentially superior advantages. The half-life of the antiATR-001 antibody was 14.4 days [11], longer than any other chemical drugs presently used. No obvious feedback activation of circulating or local RAS was observed. Additionally, long-term treatment of chronic diseases is costly, tedious, and at the population level rather unsuccessful. Monoclonal antibodies specific for host proteins have proven to be highly effective, and the movement from the passive administration of monoclonal antibodies to active vaccination against selfmolecular could provide affordable medicines and broader patient acceptance and compliance. The question today in many patient populations is not whether to block the RAS but rather how best to inhibit its activity. From a theoretical perspective, vaccination has much to recommend it as a strategy. ATRQ $\beta-001$ vaccine modulates two major axes of RAS and ameliorates STZ-induced diabetic injury with no obvious immune-mediated damages, providing a promising therapeutic method to treat diabetic nephropathy.

Acknowledgments This work was supported by the Major Research Plan of the National Natural Science Foundation of China (No. 91439207) and National Natural Science Foundation of China (Nos. 81300246, 81400314, 81270331, 31370931, 81300196, 81470494).

Conflict of interest All the authors declared no financial conflicts of interests.
Open Access This article is distributed under the terms of the Creative Commons Attribution 4.0 International License (http:// creativecommons.org/licenses/by/4.0/), which permits unrestricted use, distribution, and reproduction in any medium, provided you give appropriate credit to the original author(s) and the source, provide a link to the Creative Commons license, and indicate if changes were made.

\section{References}

1. Boyle JP, Thompson TJ, Gregg EW, Barker LE, Williamson DF (2010) Projection of the year 2050 burden of diabetes in the US adult population: dynamic modeling of incidence, mortality, and prediabetes prevalence. Popul Health Metr 8:29

2. Ribeiro-Oliveira AJ, Nogueira AI, Pereira RM, Boas WW, Dos SR, Simoes ESA (2008) The renin-angiotensin system and diabetes: an update. Vasc Health Risk Manag 4:787-803

3. Bakris GL, Williams M, Dworkin L, Elliott WJ, Epstein M, Toto R, Tuttle K, Douglas J, Hsueh W, Sowers J (2000) Preserving renal function in adults with hypertension and diabetes: a consensus approach. National Kidney Foundation Hypertension and Diabetes Executive Committees Working Group. Am J Kidney Dis 36: 646-661

4. Brenner BM, Cooper ME, de Zeeuw D, Keane WF, Mitch WE, Parving HH, Remuzzi G, Snapinn SM, Zhang Z, Shahinfar S (2001) Effects of losartan on renal and cardiovascular outcomes in patients with type 2 diabetes and nephropathy. $\mathrm{N}$ Engl J Med 345:861-869

5. Lewis EJ, Hunsicker LG, Clarke WR, Berl T, Pohl MA, Lewis JB, Ritz E, Atkins RC, Rohde R, Raz I (2001) Renoprotective effect of the angiotensin-receptor antagonist irbesartan in patients with nephropathy due to type 2 diabetes. N Engl J Med 345:851-860

6. Nankervis A, Nicholls K, Kilmartin G, Allen P, Ratnaike S, Martin FI (1998) Effects of perindopril on renal histomorphometry in diabetic subjects with microalbuminuria: a 3-year placebo-controlled biopsy study. Metabolism 47:12-15

7. Cordonnier DJ, Pinel N, Barro C, Maynard M, Zaoui P, Halimi S, Hurault DLB, Reznic Y, Simon D, Bilous RW (1999) Expansion of cortical interstitium is limited by converting enzyme inhibition in type 2 diabetic patients with glomerulosclerosis. The Diabiopsies Group. J Am Soc Nephrol 10:1253-1263

8. Haller H, Ito S, Izzo JJ, Januszewicz A, Katayama S, Menne J, Mimran A, Rabelink TJ, Ritz E, Ruilope LM et al (2011) Olmesartan for the delay or prevention of microalbuminuria in type 2 diabetes. N Engl J Med 364:907-917

9. Roscioni SS, Heerspink HJ, de Zeeuw D (2014) The effect of RAAS blockade on the progression of diabetic nephropathy. Nat Rev Nephrol 10:77-87

10. Atlas SA (2007) The renin-angiotensin aldosterone system: pathophysiological role and pharmacologic inhibition. J Manag Care Pharm 13:9-20

11. Chen X, Qiu Z, Yang S, Ding D, Chen F, Zhou Y, Wang M, Lin J, Yu X, Zhou Z et al (2013) Effectiveness and safety of a therapeutic vaccine against angiotensin II receptor type 1 in hypertensive animals. Hypertension 61:408-416

12. Zhu F, Liao YH, Li LD, Cheng M, Wei F, Wei YM, Wang M (2006) Target organ protection from a novel angiotensin II receptor (AT1) vaccine ATR12181 in spontaneously hypertensive rats. Cell Mol Immunol 3:107-114

13. Azegami T, Sasamura H, Hayashi K, Itoh H (2012) Vaccination against the angiotensin type 1 receptor for the prevention of LNAME-induced nephropathy. Hypertens Res 35:492-499 
14. Tesch GH, Allen TJ (2007) Rodent models of streptozotocininduced diabetic nephropathy. Nephrology (Carlton) 12:261-266

15. Mundel P, Shankland SJ (2002) Podocyte biology and response to injury. J Am Soc Nephrol 13:3005-3015

16. Lan HY, Chung AC (2012) TGF-beta/Smad signaling in kidney disease. Semin Nephrol 32:236-243

17. Breyer MD, Bottinger E, Brosius FC, Coffman TM, Harris RC, Heilig CW, Sharma K (2005) Mouse models of diabetic nephropathy. J Am Soc Nephrol 16:27-45

18. Santos RA, Ferreira AJ, Simoes ESA (2008) Recent advances in the angiotensin-converting enzyme 2-angiotensin(1-7)-Mas axis. Exp Physiol 93:519-527

19. Santos RA, Ferreira AJ, Verano-Braga T, Bader M (2013) Angiotensin-converting enzyme 2, angiotensin-(1-7) and Mas: new players of the renin-angiotensin system. J Endocrinol 216:R1-R17

20. Li N, Zimpelmann J, Cheng K, Wilkins JA, Burns KD (2005) The role of angiotensin converting enzyme 2 in the generation of angiotensin 1-7 by rat proximal tubules. Am J Physiol Renal Physiol 288: F353-F362

21. Soler MJ, Wysocki J, Ye M, Lloveras J, Kanwar Y, Batlle D (2007) ACE2 inhibition worsens glomerular injury in association with increased ACE expression in streptozotocin-induced diabetic mice. Kidney Int 72:614-623

22. Oudit GY, Liu GC, Zhong J, Basu R, Chow FL, Zhou J, Loibner H, Janzek E, Schuster M, Penninger JM et al (2010) Human recombinant ACE2 reduces the progression of diabetic nephropathy. Diabetes 59:529-538

23. Zhang K, Meng X, Li D, Yang J, Kong J, Hao P, Guo T, Zhang M, Zhang Y, Zhang C (2015) Angiotensin(1-7) attenuates the progression of streptozotocin-induced diabetic renal injury better than angiotensin receptor blockade. Kidney Int 87:359-369

24. Mori J, Patel VB, Ramprasath T, Alrob OA, DesAulniers J, Scholey JW, Lopaschuk GD, Oudit GY (2014) Angiotensin 1-7 mediates renoprotection against diabetic nephropathy by reducing oxidative stress, inflammation, and lipotoxicity. Am J Physiol Renal Physiol 306:F812-F821

25. Bachmann MF, Dyer MR (2004) Therapeutic vaccination for chronic diseases: a new class of drugs in sight. Nat Rev Drug Discov 3:81-88

26. Rammensee H, Bachmann J, Emmerich NP, Bachor OA, Stevanovic S (1999) SYFPEITHI: database for MHC ligands and peptide motifs. Immunogenetics 50:213-219

27. Ludwig C, Wagner R (2007) Virus-like particles-universal molecular toolboxes. Curr Opin Biotechnol 18:537-545

28. Bachmann MF, Rohrer UH, Kundig TM, Burki K, Hengartner H, Zinkernagel RM (1993) The influence of antigen organization on B cell responsiveness. Science 262:1448-1451

29. Ambuhl PM, Tissot AC, Fulurija A, Maurer P, Nussberger J, Sabat R, Nief V, Schellekens C, Sladko K, Roubicek K et al (2007) A vaccine for hypertension based on virus-like particles: preclinical efficacy and phase I safety and immunogenicity. J Hypertens 25: 63-72

30. Tissot AC, Maurer P, Nussberger J, Sabat R, Pfister T, Ignatenko S, Volk HD, Stocker H, Muller P, Jennings GT et al (2008) Effect of immunisation against angiotensin II with CYT006-AngQb on ambulatory blood pressure: a double-blind, randomised, placebocontrolled phase IIa study. Lancet 371:821-827 